\title{
Predicting Revision Following In Situ Ulnar Nerve Decompression for Patients With Idiopathic Cubital Tunnel Syndrome.
}

\author{
Michael P. Gaspar \\ The Philadelphia Hand Center; Thomas Jefferson University \\ Patrick M. Kane \\ Thomas Jefferson University; Philadelphia Hand Center \\ Dechporn Putthiwara \\ Philadelphia Hand Center \\ Sidney M. Jacoby \\ Thomas Jefferson University; Philadelphia Hand Center \\ Aoltew thistearkąaditional works at: https://jdc.jefferson.edu/orthofp \\ Thomas Jefferson University; Philadelphia Hand Center \\ Part of the Orthopedics Commons, and the Surgery Commons

\section{Let us know how access to this document benefits you}

\section{Recommended Citation}

Gaspar, Michael P.; Kane, Patrick M.; Putthiwara, Dechporn; Jacoby, Sidney M.; and Osterman, A. Lee, "Predicting Revision Following In Situ Ulnar Nerve Decompression for Patients With Idiopathic Cubital Tunnel Syndrome." (2016). Department of Orthopaedic Surgery Faculty Papers. Paper 90.

https://jdc.jefferson.edu/orthofp/90

This Article is brought to you for free and open access by the Jefferson Digital Commons. The Jefferson Digital Commons is a service of Thomas Jefferson University's Center for Teaching and Learning (CTL). The Commons is a showcase for Jefferson books and journals, peer-reviewed scholarly publications, unique historical collections from the University archives, and teaching tools. The Jefferson Digital Commons allows researchers and interested readers anywhere in the world to learn about and keep up to date with Jefferson scholarship. This article has been accepted for inclusion in Department of Orthopaedic Surgery Faculty Papers by an authorized administrator of the Jefferson Digital Commons. For more information, please contact: JeffersonDigitalCommons@jefferson.edu. 


\section{ABSTRACT}

2 PURPOSE

3 To determine the incidence of revision and potential risk factors for needing

4 revision surgery following in situ ulnar nerve decompression for patients with

5 idiopathic cubital tunnel syndrome (CuTS).

6

7 METHODS

8 We conducted a retrospective chart review of all patients treated at one specialty

9 hand center with an open in situ ulnar nerve decompression for idiopathic CuTS

10 from January 2006 through December 2010. Revision incidence was determined by

11 identifying patients who underwent additional surgeries for recurrent or persistent

12 ulnar nerve symptoms. Bivariate analysis was performed to determine which

13 variables had a significant influence on the need for revision surgery.

\section{$15 \quad$ RESULTS}

16 Revision surgery was required in $3.2 \%$ (7 of 216 ) of all cases. Age of less than 50

17 years at the time of index decompression was the lone significant predictor of need

18 for revision surgery. Other patient factors, including sex, diabetes, smoking history,

19 and worker compensation status were not predictive of the need for revision

20 surgery. Disease-specific variables including nerve conduction velocities, McGowan

21 grading, and predominant symptom type were also not predictive of revision. 


\section{CONCLUSIONS}

24 For patients with idiopathic cubital tunnel syndrome, the risk of revision surgery

25 following in situ ulnar nerve decompression is low. However, this risk was

26 increased in patients who were younger than 50 years of age at the time of the index

27 procedure. The findings of this study suggest that, in the absence of underlying

28 elbow arthritis or prior elbow trauma, in situ ulnar nerve decompression is an

29 effective, minimal-risk option for the initial surgical treatment of CuTS.

30

31 LEVEL OF EVIDENCE

32 Prognostic Level III

33 


\section{INTRODUCTION}

35 Cubital tunnel syndrome (CuTS) is second only to carpal tunnel syndrome in

36 incidence among compression neuropathies of the upper extremity. [1-3] Despite its

37 commonality, there is no established consensus regarding the optimal surgical

38 treatment. This is evidenced by a wide range of surgical options including in situ

39 decompression, medial epicondylectomy and subcutaneous, intramuscular or

40 submuscular transposition of the ulnar nerve. Additionally, in recent years surgeons

41 have also advocated for endoscopic or minimal-incision release of the ulnar nerve,

42 with or without transposition, to further minimize soft tissue trauma and potential

43 vascular insult to the nerve, while allowing for faster recovery, thus further

44 expanding the number of treatment options. [4-6]

46 Technique selection can depend on a variety of factors including surgeon

47 preference, patient anatomy, patient desires, underlying pathology, and

48 complication rates. Transposition, for example, often requires extensive dissection

49 around the nerve, which may compromise its extrinsic vascular supply. Thus, it may

50 be contraindicated in patients with diabetes for instance who may have a tenuous

51 vascular system at the level of the cubital tunnel. [7, 8] In addition, with an

52 increasing focus on healthcare economics in the United States, the relative cost-

53 effectiveness of different treatment options for CuTS may progressively factor into

54 surgical decision-making, thus potentially clouding the treatment decision even

55 further. [9-11] 
57 Generally, in situ decompression offers the least invasive surgical option but may

58 increase the risk of revision surgery. [12,13] A recent study found that prior history

59 of trauma around the elbow was a notable predictor of need for revision after in situ

60 decompression of the ulnar nerve, while other postulated factors including patient

61 age had no effect. [14] However, risk factors for revision in patients with idiopathic

62 CuTS, that is, those without an underlying traumatic, arthritic, or other pre-

63 disposing etiology, remain unclear. As revision surgery yields inferior outcomes

64 versus primary surgery for CuTS, information on risk factors leading to revision in

65 these patients with idiopathic CuTS could provide a valuable addition to the overall

66 treatment algorithm. [15]

67 The purpose of this study was to determine the incidence of needed revision after in

68 situ ulnar nerve decompression for patients with idiopathic CuTS and to investigate

69 which patient risk factor(s) may contribute to an increased likelihood of needing

70 revision.

72 MATERIALS and METHODS

73 This study was approved by our institutional review board. Using our departmental

74 electronic billing database search for Current Procedural Terminology (American

75 Medical Association, Chicago, IL, USA) code 64718 (surgery on ulnar nerve at

76 elbow), we identified all patients who had undergone in situ ulnar nerve

77 decompression surgery from January 2006 through December 2010. Patients who 
78 demonstrated intraoperative subluxation of the ulnar nerve following in situ

79 decompression were excluded, as these patients subsequently underwent either

80 anterior transposition of the ulnar nerve or medial epicondylectomy. Patients were

81 also excluded if they underwent in situ ulnar nerve decompression for reasons other

82 than treatment of CuTS symptoms (e.g., prophylactic release performed in

83 conjunction with elbow arthroplasty or fracture fixation) or had previously

84 undergone operative treatment for CuTS. In addition, patients with a prior history of

85 fracture or trauma at the elbow were excluded, as were those with a history of

86 degenerative, post-traumatic, or inflammatory arthritis at the elbow. However,

87 patients with a known history of inflammatory or systemic arthritis without

88 evidence of local arthritic changes at the surgical elbow were not excluded. Finally,

89 patients with less than 6 months of follow-up at our institution were excluded from

90 data analysis unless a revision surgery occurred in that time interval. Records for

91 those patients with less than 6 months of follow-up were reviewed in an effort to

92 predict their clinical course. In addition, attempts were made to contact those

93 patients via telephone with the goal of identifying any patients that may have had

94 additional surgery performed elsewhere.

\section{Diagnostic workup}

97 Patients seen at our institution are generally evaluated by the treating surgeon prior

98 to obtaining additional studies, including imaging or electrodiagnostic testing.

99 Exceptions to this practice typically only occur in patients who are seen at our

100 institution for a second opinion and have already undergone electrodiagnostic 
101 testing prior to presentation. During initial evaluation, a comprehensive clinical

102 examination, including disease-specific tests and provocative maneuvers, is

103 performed. This includes 2 point-discrimination, vibratory discrimination testing,

104 comparative grip strength testing, cross-finger testing, Froment sign, Tinel sign,

105 elbow flexion-compression test, and testing for nerve mobility. When a patient is

106 suspected of having CuTS based on clinical history and physical examination,

107 standard elbow radiographs are routinely obtained to rule out contributory bony

108 abnormalities or deformities in addition to electrodiagnostic testing. Nerve

109 conduction tests are considered abnormal if conduction velocity across the affected

110 elbow is less than 50 meters per second or is decreased by more than 10 meters per

111 second across the elbow. The diagnosis of CuTS is based on clinical findings in

112 conjunction with nerve testing results.

113

114 Additionally, effort is made to elucidate any nerve symptoms not originating at the

115 elbow, such as proximally based cervical pathology or distal compression of the

116 ulnar and median nerves at the wrist. When the diagnostic workup suggests

117 pathology at those distal sites, it is not uncommon in our practice to perform

118 concomitant release of the ulnar and median nerves at the Guyon canal and the

119 carpal tunnel. However, for those patients with findings of ipsilateral cervical

120 radiculopathy, the cervical pathology is generally addressed prior to any operative

121 management of CuTS-related symptoms.

\section{Operative indications}


124 Indications for primary in situ decompression generally involve nerve symptoms

125 consistent with CuTS that have failed a trial of conservative management, have

126 positive electrodiagnostic findings, and have a stable ulnar nerve. At our institution,

127 ulnar nerve hypermobility, manifested as nerve subluxation or dislocation during

128 preoperative or intraoperative assessment, is considered a contraindication to

129 performing in situ decompression alone. Thus, when such hypermobility is noted,

130 alternative surgical options such as anterior ulnar nerve transposition or medial

131 epicondylectomy are considered.

132

133 The decision to operate on patients with CuTS in the revision setting is a joint-

134 agreement between the patient and surgeon. Although this is normally approached

135 on a case-by-case basis, the typical scenario involves persistent or incomplete-

136 resolution of symptoms compared to preoperatively. Workup for recurrent or

137 persistent CuTS is largely the same as in primary CuTS described above.

\section{Surgical technique and postoperative protocol}

140 All surgeries were performed by one of 8, fellowship-trained orthopedic hand

141 surgeons. A posteromedial incision measuring 5 to 10 centimeters centered about

142 the epicondylar groove is used for exposure. As the incision is carried

143 subcutaneously, care is taken to identify and protect branches of the medial

144 antebrachial cutaneous nerve. Upon identification of the ulnar nerve,

145 decompression is performed via surgical release of the Osborne ligament and fascia

146 overlying the flexor carpi ulnaris with blunt dissection carried roughly 8 
147 centimeters proximally to the level of the arcade of Struther. In those patients found

148 to have an anconeus epitrochlearis, the anomalous muscle is generally split or

149 excised depending on its involvement in compression of the ulnar nerve. Care is

150 taken to avoid circumferential dissection around the nerve to preserve its vascular

151 supply. Following release, the elbow is taken through its full range-of-motion to

152 confirm stability of the ulnar nerve. Postoperatively, the limb is placed in a well-

153 padded posterior long-arm orthosis with the elbow positioned in approximately 70

154 degrees of flexion. Active range-of-motion is typically initiated subsequent to the

155 first postoperative visit one week following surgery. Nerve conduction testing is not

156 routinely performed postoperatively except in cases of persistent, recurrent, or

157 worsening symptoms.

159 Data collection and statistical analysis

160 For those patients satisfying inclusion in the study, demographic, medical, and

161 surgical data were obtained from departmental records. We defined our primary

162 outcome of interest to be revision cubital tunnel surgery performed after in situ

163 ulnar nerve decompression. Thus any patients, who at the time of data analysis had

164 not had revision surgery, were designated to the control cohort. Bivariate analysis

165 was performed for categorical variables of sex, diabetes history, smoking history,

166 presence of bilateral symptoms, predominant preoperative symptom, modified pre-

167 and postoperative modified McGowan grade, concomitant surgery, and worker

168 compensation status using Chi-square or Fisher exact testing. Continuous variables

169 recorded preoperatively including symptom duration, body mass index (BMI), and 
170 nerve conduction velocity (NCV), were compared using Student t-test or Mann-

171 Whitney $U$ test. Age was analyzed as both a categorical variable (less than 50 years

172 versus greater-than-or-equal-to 50 years) and as a continuous variable.

\section{$174 \quad \underline{\text { RESULTS }}$}

175 A total of 216 elbows in 201 patients satisfied inclusion in this study. (See Figure 1)

176 The mean age at the time of surgery for all 216 cases was $53+/-14$ years, with

177 mean follow-up duration of $22+/-21$ months. Continuous and categorical

178 demographic variables of the entire study cohort are represented in Tables 1 and 2, 179 respectively.

181 Revision surgery was required in 7 (3.2\%) cases, with the first revision occurring at 182 a median interval of 10 months from the index surgery (range 3 to 59 months). Five

183 of those patients were revised with anterior subcutaneous transposition, one with

184 submuscular transposition, and one with intramuscular transposition. Two patients

185 required more than one revision for persistent or recurrence of symptoms.

186 Treatment course and demographic characteristics of those patients requiring

187 revision surgery are outlined in Table 3.

189 Bivariate analysis

190 Younger age had a statistically significant effect on need for revision surgery when

191 analyzed as a continuous variable, mean age non-revised $=53+/-14$ years versus

192 revised $=43+/-7$ years; $P=0.009$, (see Table 1 ) and as a categorical variable (age $\geq$ 
19350 years vs. age $<50$ years; Fisher exact test, $P=0.002$, see Table 2). The duration of

194 preoperative symptoms in the revised cohort was roughly double that of the

195 controls, although this association only approached statistical significance $(12+/-$

19611 months versus $26+/-17$ months; $P=0.08$, Table 1 ). Patient sex, diabetes history,

197 smoking history, predominant symptom at this time of surgery, modified McGowan

198 grade, concomitant surgery, worker compensation status, body mass index, and

199 ulnar nerve conduction velocity values were not statistically different between

200 those patients requiring revision and those who did not. Figure 2 illustrates the

201 change in modified McGowan grade for the entire study cohort. Tables 1 and 2 detail

202 the respective relationships of continuous and categorical variables and the need for

203 revision surgery.

204

205 Subjective and validated outcomes

206 No patients reported worsening of their symptoms following ulnar nerve in situ

207 decompression compared to preoperatively. Of the 209 patients who did not

208 undergo revision surgery, 3 patients complained of persistent sensory symptoms

209 and were offered revision surgery, but they declined. A fourth patient reported

210 recurrence of her symptoms and expressed desire to undergo revision surgery, but

211 she was subsequently lost to follow-up. Multiple attempts to contact that patient via

212 telephone were unsuccessful. The remaining 205 patients reported subjective

213 improvement and general satisfaction following their operation. Table 3 details the

214 treatment course of the revision cohort. 
217 DISCUSSION

218 Selecting the optimal surgical treatment plan for patients with idiopathic cubital

219 tunnel syndrome remains a difficult task. Though numerous studies have explored

220 differences in outcomes among the various surgical options, results have often been

221 inconclusive, and at times, contradictory. [3, 16-18]

223 Need for revision surgery is a particularly important outcome to investigate, as it

224 not only represents a sub-optimal clinical result but has important economic

225 considerations as well. With a lack of high quality, adequately powered prospective

226 randomized-control trials comparing the multitude of surgical options for CuTS,

227 cost-effectiveness and decision analyses may afford clinicians a useful tool for

228 comparisons when real-world studies fall short or may simply be impractical. [9, 11,

229 19] A decision analysis study concluded that in situ decompression of the ulnar

230 nerve had the highest utility of 4 tested surgical procedures, while medial

231 epicondylectomy fared worst. [10] These results were later supported by Song et al,

232 who explored the same four surgical treatments for CuTS and found that in situ

233 decompression to be superior to the other options in cost-effectiveness. [11] Both

234 studies used literature available at the time to account for expected incidences of

235 complications and revision for each of the 4 surgical treatments examined. In a

236 randomized-control trial comparing ulnar nerve in situ decompression with

237 anterior subcutaneous transposition, Bartels et al found in situ decompression to be 
238 superior from a cost perspective, while also demonstrating a lower incidence of

239 complications. [3, 9]

241 Despite the findings of these studies supporting in situ decompression as a first-

242 option for CuTS, the question remains as to which patients are best suited for this

243 versus other surgical options for CuTS, particularly in regards to circumventing the

244 need for revision surgery. Determining which patients are most likely to need

245 revision surgery after initial decompression could be equally as valuable as the

246 previously mentioned cost and decision-based analyses in avoiding the medical and

247 economic costs associated with a second surgery. Krogue and colleagues studied

248 factors leading to revision after in situ ulnar nerve decompression for CuTS and

249 found that a prior history of elbow trauma was the most notable variable predicting

250 the need for revision surgery after simple decompression. [14] In light of those

251 findings, we determined that further investigation into risk factors leading to

252 revision for patients with idiopathic would provide additional information to

253 surgeons contemplating surgical options for CuTS.

255 In this study, we report an overall revision incidence of $3.2 \%$, which is lower than

256 previous studies of in situ decompression. At least one potential factor for this

257 difference is the exclusion of patients with traumatic or arthritic etiology. However,

258 this is not completely unlike a previous study by Goldfarb et al, who excluded

259 patients with elbow arthritis, medial epicondylitis, and ulnar nerve subluxation, and 
260 reported a revision incidence of 7\%. [12] When Krogue et al implemented even less

261 stringent inclusion criteria, they reported a revision incidence of 19\%. [14] Taken

262 together, these 3 studies suggest that, in the absence of both traumatic and arthritic

263 conditions, simple in situ decompression of the ulnar nerve for CuTS has an low

264 incidence of revision. A comparative overview of the these studies is included in

265 Table 4.

266

267 Our study also provides statistically significant evidence that younger age is a risk

268 factor for needing revision surgery in these patients. Although the clinical meanaing

269 of this finding is less clear, the relationship of younger age as a pre-disposing factor

270 to complications after in situ decompression is not novel. Murata et al demonstrated

271 younger age to be predictive of increased incidence of ulnar nerve dislocation, as

272 simulated intra-operatively by placing patients' elbows in full-flexion after ulnar

273 nerve decompression. [20] Theysuggested that anatomical differences in the size of

274 the medial epicondyle and the shape of the ulnar groove played a role in the higher

275 nerve dislocation incidence in younger patients. All elbows in our study were

276 confirmed to have a ulnar nerve that neither subluxed or dislocated when tested

277 intra-operatively after release had been performed during the index procedure.

278 However, of the 7 cases requiring revision, 4 were noted to have a subluxating ulnar

279 nerve at the time of revision surgery. None of these 4 patients was noted to have

280 nerve instability in their latest physical examination prior to undergoing revision. It

281 remains unclear as to the mechanism by which a confirmed stable ulnar nerve 
282 would later become unstable without any further intervention. In addition, we were

283 unable to account for the fact that these nerves appeared stable during examination

284 and only after surgical re-exposure were they unstable. We speculate that perhaps

285 some of the soft tissue and scarring that was released to gain exposure at the time of

286 revision surgery may have also had a tethering effect on the nerve. Regardless of the

287 means through which younger age predicts a higher revision incidence following in

288 situ decompression for treatment of CuTS, these findings suggest a consideration for

289 surgeons to discuss with younger patients seeking operative treatment for CuTS.

291 This study has limitations. Its retrospective nature required that we rely strictly on

292 medical records, which were not always complete and could be subject to

293 interpretation. In addition, though we only included patients who had at least 6

294 months of follow-up at our institution, there is potential for bias if any patients

295 sought care involving revision surgery elsewhere after that initial period. We sought

296 to minimize this possibility by attempting to reach patients via telephone while also

297 reviewing records for those patients to predict which, if any, would be likely to seek

298 care elsewhere. We were unable to contact over one-third of those patients with less

299 than 6 months of follow-up (see Figure 1). Furthermore, relying solely on clinical

300 documentation to speculate on this type of information is imperfect. Lastly, while

301 our specific aim was to investigate risk factors specific to idiopathic CuTS, exclusion

302 of patients with post-traumatic or arthritic etiologies may have led to us to

303 underestimate a clinically relevant revision incidence. 
305 Despite these limitations, our results may be useful in establishing a treatment

306 algorithm for uncomplicated idiopathic CuTS. In particular, for patients confirmed

307 to have CuTS without arthritis or history of trauma to the involved elbow, our

308 findings strongly support in situ decompression as a reliable, first-line surgical

309 treatment option. The risk of revision increased somewhat in patients younger than

31050 years of age, though the underlying mechanism of this relationship remains

311 unclear.

312 


\section{REFERENCES}

314 1. Shuman S, Osterman AL, Bora FW. Compression neuropathies. Semin Neurol. 3151987 Mar;7(1):76-87. Review.

316 2. Karthik K, Nanda R, Storey S, Stothard J. Severe ulnar nerve entrapment at 317 the elbow: functional outcome after minimally invasive in situ

318 decompression. J Hand Surg Eur Vol. 2012;37(2):115-122.

319 3. Bartels RHM a, Verhagen WIM, Van Der Wilt GJ, Meulstee J, Van Rossum LGM, 320 Grotenhuis J a. Prospective randomized controlled study comparing simple 321 decompression versus anterior subcutaneous transposition for idiopathic 322 neuropathy of the ulnar nerve at the elbow: Part 1. Neurosurgery. $323 \quad 2005 ; 56(3): 522-529$.

324 4. Morse LP, Hons B, Mcguire DT, Ortho FC, Bain GI, A F a O. Endoscopic Ulnar 325 Nerve Release and Transposition. Tech Hand Up Extrem Surg. 2014;18(1):10$326 \quad 14$.

327 5. Zajonc H, Momeni A. Endoscopic release of the cubital tunnel. Hand Clin. $328 \quad 2014 ; 30(1): 55-62$.

329 6. Adkinson JM, Chung KC. Minimal-incision in situ ulnar nerve decompression 330 at the elbow. Hand Clin. 2014;30(1):63-70.

331 7. Nakamura K, Uchiyama S, Ido Y, et al. The effect of vascular pedicle 332 preservation on blood flow and clinical outcome following ulnar nerve 333 transposition. J Hand Surg Am. 2014;39(2):291-302.

334 8. Ogata K, Manske PR, Lesker PA. The effect of surgical dissection on regional 335 blood flow to the ulnar nerve in the cubital tunnel. Clin Orthop Relat Res. 336 1985; 193(Mar):195-198. 
337 9. Bartels RHM a, Termeer EH, Van Der Wilt GJ, et al. Simple decompression or 338 anterior subcutaneous transposition for ulnar neuropathy at the elbow: A 339 cost-minimization analysis - Part 2. Neurosurgery. 2005;56(3):531-535.

340 10. Brauer CA, Graham B. The surgical treatment of cubital tunnel syndrome: a 341 decision analysis. J Hand Surg Eur Vol. 2007;32(6):654-662.

342 11. Song JW, Chung KC, Prosser LA. Treatment of ulnar neuropathy at the elbow: 343 cost-utility analysis. J Hand Surg Am. 2012 Aug;37(8):1617-1629.e3.

344 12. Goldfarb C, Sutter M, Martens E, Manske P. Incidence of Re-Operation and 345 Subjective Outcome Following in Situ Decompression of the Ulnar Nerve J 346 Hand Surg Eur Vol. 2012;2009(3):379-383.

347 13. LeRoux P, Ensign T, Burchiel K. Surgical Decompression without 348 Transposition for Ulnar Neuropathy: Factors Determining Outcome. $349 \quad$ Neurosurgery. 1990;27(5):709-714.

350 14. Krogue JD, Aleem AW, Osei D a., Goldfarb C a., Calfee RP. Predictors of 351 surgical revision after in situ decompression of the ulnar nerve. J Shoulder $352 \quad$ Elbow Surg. 2015;24(4):634-639.

353 15. Aleem AW, Krogue JD, Calfee RP. Outcomes of Revision Surgery for Cubital 354 Tunnel Syndrome. J Hand Surg Am. 2014;39(11):2141-2149.

355 16. Charles YP, Coulet B, Rouzaud JC, Daures JP, Chammas M. Comparative 356 Clinical Outcomes of Submuscular and Subcutaneous Transposition of the 357 358

359 17. Macadam SA., Gandhi R, Bezuhly M, Lefaivre KA. Simple Decompression 360 Versus Anterior Subcutaneous and Submuscular Transposition of the Ulnar 
Nerve for Cubital Tunnel Syndrome: A Meta-Analysis. J Hand Surg Am. 2008;33(8):1-12.

363 18. Mitsionis GI, Manoudis GN, Paschos NK, Korompilias AV, Beris AE.

364 Comparative study of surgical treatment of ulnar nerve compression at the elbow. J Shoulder Elbow Surg. 2010 Jun;19(4):513-9.

366 19. Hill SR. Cost-effectiveness analysis for clinicians. BMC Med. 2012;10(1):10.

367 20. Murata K, Omokawa S, Shimizu T, Nakanishi Y, Kawamura K, Yajima H, 368 Tanaka Y. Risk factors for dislocation of the ulnar nerve after simple 369 decompression for cubital tunnel syndrome. Hand Surg. 2014;19(1):13-8. 


\section{FIGURES}

372 Figure 1. Flow chart of inclusion and exclusion criteria applied to potential study

373 subjects.

374

291 cases in

271 patients

In-situ ulnar nerve

decompression for CuTs

\section{3 cases in}

38 patients

History of elbow trauma and/or arthritis

248 cases in

233 patients

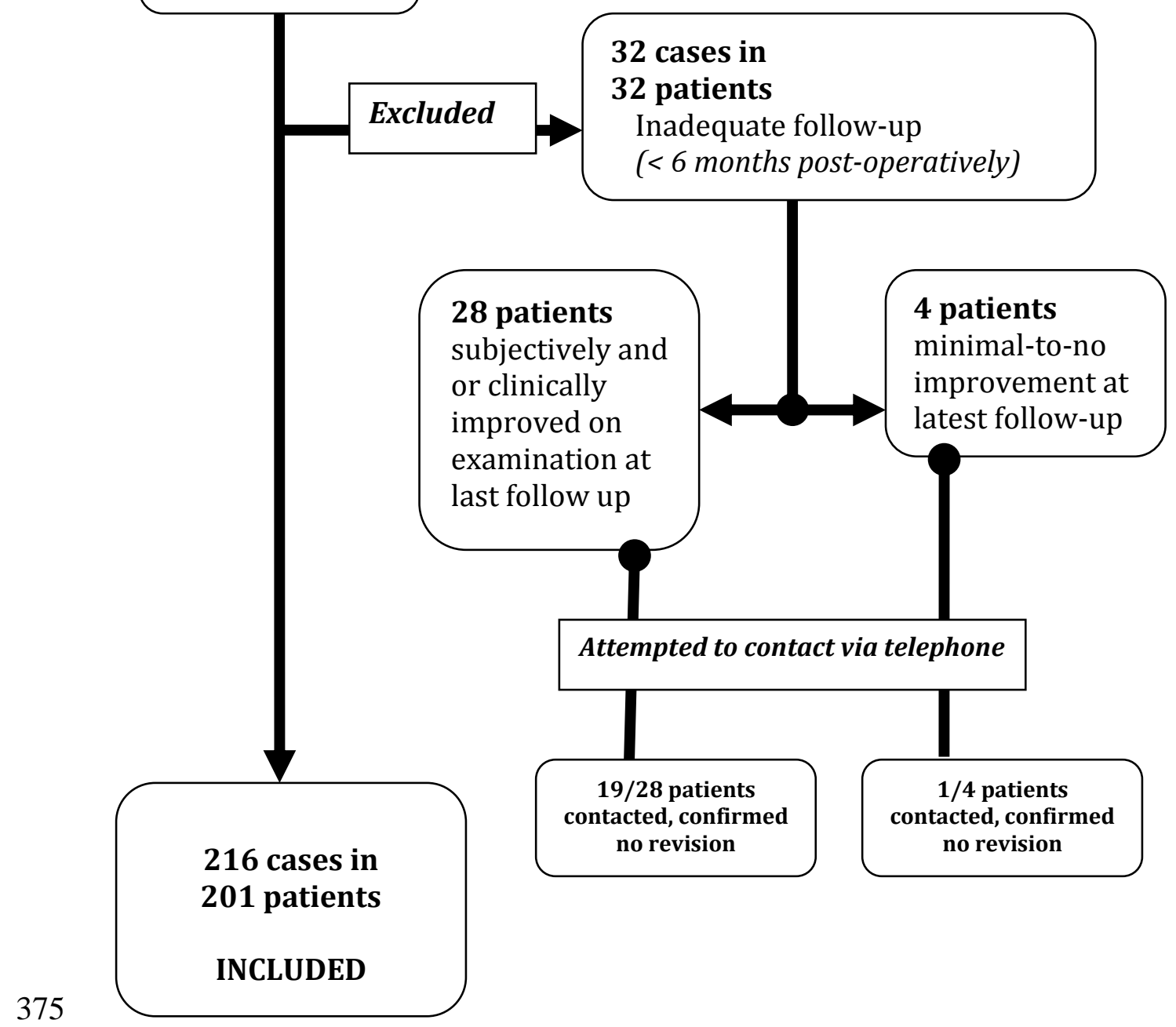


376 Figure 2. Graphical representation of change between pre- to postoperative

377 Modified McGowan grade. Aside from 2 patients with preoperative grade of IIa who 378 improved to normal postoperatively (thick dashed arrow), all other patients either 379 improved by one grade (solid arrow) or remained the same (dotted arrow).

Preoperative Modified McGowan Grade
Postoperative Modified McGowan Grade

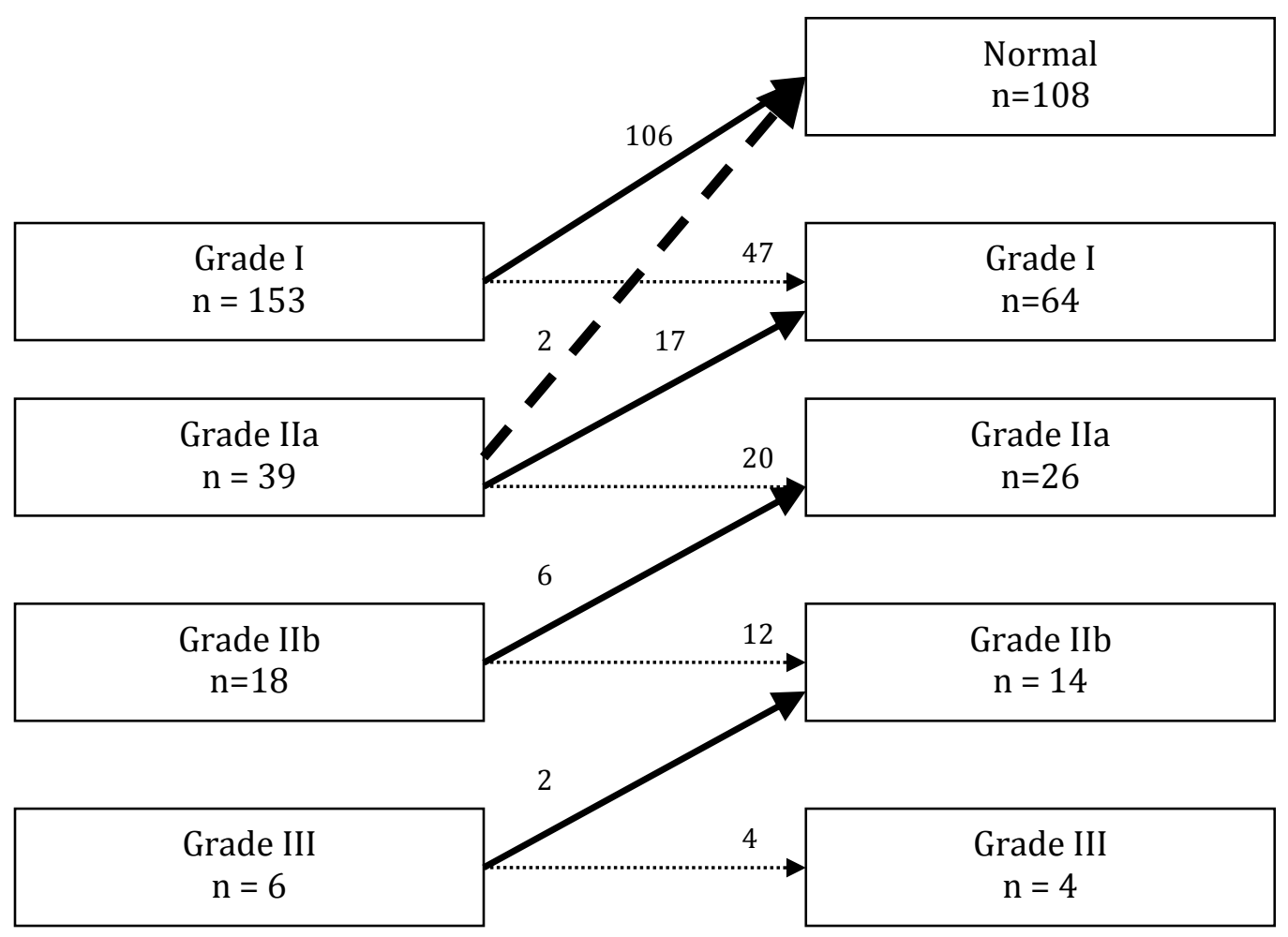

No improvement Improvement by 1 grade Improvement by 2 grades

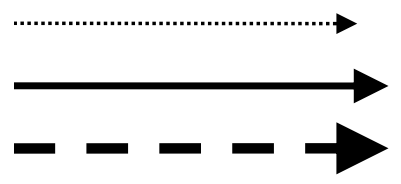

